University of Texas at El Paso

ScholarWorks@UTEP

$5-2003$

\title{
Robust Methodology for Characterizing System Response to Damage: Approach Based on Partial Order
}

\author{
Paul J. Tanenbaum \\ Carlos de la Mora \\ Piotr Wojciechowski \\ Olga Kosheleva \\ The University of Texas at El Paso, olgak@utep.edu \\ Vladik Kreinovich \\ The University of Texas at El Paso, vladik@utep.edu \\ Follow this and additional works at: https://scholarworks.utep.edu/cs_techrep \\ Pproxto a \\ Comments: \\ Technical Report: UTEP-CS-03-11a \\ Published in: Ivan Lirkov, Svetozar Margenov, Jerzy Wasniewski, and Plamen Yalamov (eds.), \\ Large-Scale Scientific Computing, Proceedings of the 4th International Conference LSSC'2003, \\ Sozopol, Bulgaria, June 4-8, 2003, Springer Lecture Notes in Computer Science, 2004, Vol. 2907, \\ pp. 238-245.
}

\section{Recommended Citation}

Tanenbaum, Paul J.; de la Mora, Carlos; Wojciechowski, Piotr; Kosheleva, Olga; Kreinovich, Vladik; Starks, Scott A.; and Kuzminykh, Alexandr V., "Robust Methodology for Characterizing System Response to Damage: Approach Based on Partial Order" (2003). Departmental Technical Reports (CS). 286.

https://scholarworks.utep.edu/cs_techrep/286

This Article is brought to you for free and open access by the Computer Science at ScholarWorks@UTEP. It has been accepted for inclusion in Departmental Technical Reports (CS) by an authorized administrator of ScholarWorks@UTEP. For more information, please contact Iweber@utep.edu. 


\section{Authors}

Paul J. Tanenbaum, Carlos de la Mora, Piotr Wojciechowski, Olga Kosheleva, Vladik Kreinovich, Scott A. Starks, and Alexandr V. Kuzminykh 


\title{
Robust Methodology for Characterizing System Response to Damage: Approach Based on Partial Order
}

\author{
Paul J. Tanenbaum ${ }^{1}$, Carlos de la Mora ${ }^{2}$, Piotr Wojciechowski ${ }^{2}$, \\ Olga Kosheleva ${ }^{2}$, Vladik Kreinovich ${ }^{2}$, Scott A. Starks ${ }^{2}$, and \\ Alexandr V. Kuzminykh ${ }^{3}$ \\ 1 U.S. Army Research Laboratory, Attn: AMSRL-SL-BE \\ Aberdeen Proving Ground, MD 21005-5068, USA \\ pjt@arl.army.mil \\ 2 NASA Pan-American Center for Earth \\ and Environmental Studies (PACES) \\ University of Texas at El Paso, El Paso, TX 79968, USA \\ contact email vladik@cs.utep.edu \\ 3 Department of Mathematics, Purdue University \\ W. Lafayette, IN 47907 , USA \\ akuzmin@math . purdue.edu
}

\begin{abstract}
To describe the response of engineering complex systems to various damage mechanics, engineers have traditionally use numbervalued utilities to describe the results of different possible outcomes, and (number-valued) probabilities (often, subjective probabilities) to describe the relative frequency of different outcomes. This description is based on the assumption that experts can always make a definite preference between two possible outcomes, i.e., that the set of all outcomes is linearly (totally) ordered. In practice, experts often cannot make a choice, their preference is only a partial order. In this paper, we describe a new approach based on partial order.
\end{abstract}

\section{Introduction}

To describe the response of engineering complex systems to various damage mechanics, engineers have traditionally use number-valued utilities to describe the results of different possible outcomes, and (number-valued) probabilities (often, subjective probabilities) to describe the relative frequency of different outcomes. This description is based on the assumption that experts can always make a definite preference between two possible outcomes, i.e., that the set of all outcomes is linearly (totally) ordered.

In practice, experts often cannot make a choice, their preference is only a partial order. For example, one of the main criteria for a tank design is that the tank retain most of its functionality after a direct hit. It is, however, difficult to describe the remaining functionality (utility) by a single numerical value. Some designs place more protection on the tank's weapons; so, when a tank is hit, it 
will retain most of its shooting capabilities-but its movement abilities may be severely damaged. In other designs, there is more protection on the engine and on the tracks, so the tank may lose its shooting abilities-but keep its ability to move fast. It is difficult to make a definite selection because depending on the battlefield situation, different designs will work best: in active defense, an immobilized tank is still a valuable shooting force-and thus of much larger value than a moving tank with no shooting capabilities-while in a fast long-distance attack, an immobilized tank is practically useless.

In such situations, when we use traditional totally-ordered techniques, we thus force an expert to make a more or less arbitrary choice between two difficultto-compare outcomes; different choices lead to different numerical values of utilities and probabilities and-as a result-to different decisions.

It is therefore desirable to come up with a decision-making procedure that would be robust, i.e., that would only depend on the actual expert choices.

The main reason why the traditional number-valued approach to decision making is widely used is that this approach is based on a solid foundation: there are axioms, principles that - if true - uniquely lead to probabilities, utilities, and corresponding techniques for decision making. Most of these principles are pretty reasonable, with the exception of one: that the corresponding ordering of alternatives is "total" ("linear"). Traditional decision theory (see, e.g., $[2,3]$ ) is based on the assumption that a person whose preferences we want to describe can always (linearly) order his preferences, i.e., that for every two alternatives $a$ and $a^{\prime}$, he can decide:

- whether $a$ is better than $a^{\prime}$ (we will denote it by $a^{\prime} \prec a$ );

- or whether $a^{\prime}$ is better than $a\left(a \prec a^{\prime}\right)$;

- or whether $a$ and $a^{\prime}$ are (for this person) of the same quality (we will denote it by $a \sim a^{\prime}$ ).

A similar assumption (often implicit) underlies the traditional description of degrees of belief ("subjective probabilities") by numbers from the interval $[0,1]$.

As we have mentioned, in real life, an expert may not be able to always compare two different alternatives. In this paper, we provide an exact description of decision making under partial ordering of alternatives. In turns out that in general, the uncertainty of each situation is characterized not by a scalar linearly ordered quantity (probability), but by a matrix-type partially ordered quantity (ordered operator).

Important particular cases are interval-valued probabilities and more general algebraic structures described by S. Markov and his group; see, e.g., [8], [9].

Our results were partially published in [6] and [7].

\section{Traditional Utility Theory: A Brief Reminder}

In this section, we will mainly follow standard definitions (see, e.g., $[2,3]$ ), but we will not always follow them exactly: in some cases, we will slightly rephrase these definitions (without changing their mathematical contents) so as to make the following transition to partially ordered preferences as clear as possible. 
Definition 1. Let $\mathcal{A}$ be a set; this set will be called the set of alternatives (or the set of pure alternatives). By a lottery on $\mathcal{A}$ we understand a a probability measure on $\mathcal{A}$ with finite support.

In other words, a lottery is a pair $\langle A, p\rangle$, where $A=\left\{a_{1}, \ldots, a_{n}\right\} \subseteq \mathcal{A}$ is a finite subset of $\mathcal{A}$, and $p$ is a mapping $p: A \rightarrow[0,1]$ for which $p\left(a_{i}\right) \geq 0$ and $\sum p\left(a_{i}\right)=1$. A lottery will also denoted as $p\left(a_{1}\right) \cdot a_{1}+\ldots+p\left(a_{n}\right) \cdot a_{n}$. We do not consider lotteries with infinite numbers of alternatives, because every real-life randomizing device, be it a dice or a computer-based random number generator, produces only finitely many possibilities.

The set of lotteries will be denoted by $L$. On this set $L$, we can naturally define an operation of probability combination as a convex combination of the corresponding probability measures: namely, if we have $m$ values $q_{1}, \ldots, q_{m} \in$ $[0,1]$ with $\sum q_{j}=1$, and $m$ lotteries $\ell_{j}=\left\langle A_{j}, p_{j}\right\rangle$, then we can define the probability combination $\ell=q_{1} \cdot \ell_{1}+\ldots+q_{m} \cdot \ell_{m}$ as a lottery $\ell=\langle A, p\rangle$ with $A=\cup A_{j}$ and $p(a)=\sum q_{j} \cdot p_{j}(a)$, where the sum is taken over all $j$ for which $a \in A_{j}$.

Definition 2. Let $\mathcal{A}$ be a set, and let $L$ be the set of all lotteries over $\mathcal{A}$. By a preference relation, we mean a pair $\langle\prec, \sim\rangle$, where $\prec$ is a (strict) order on $L, \sim$ is an equivalence relation on $L$, and for every $\ell, \ell^{\prime}, \ell^{\prime \prime} \in L$ and every $p \in(0,1)$, the following conditions hold:

1. if $\ell \sim \ell^{\prime}$ and $\ell^{\prime} \prec \ell^{\prime \prime}$, then $\ell \prec \ell^{\prime \prime}$;

2. if $\ell \prec \ell^{\prime}$ and $\ell^{\prime} \sim \ell^{\prime \prime}$, then $\ell \prec \ell^{\prime \prime}$;

3. if $\ell \prec \ell^{\prime}$, then $p \cdot \ell+(1-p) \cdot \ell^{\prime \prime} \prec p \cdot \ell^{\prime}+(1-p) \cdot \ell^{\prime \prime}$;

4. if $p \cdot \ell+(1-p) \cdot \ell^{\prime \prime} \prec p \cdot \ell^{\prime}+(1-p) \cdot \ell^{\prime \prime}$, then $\ell \prec \ell^{\prime}$;

5. if $\ell \sim \ell^{\prime}$, then $p \cdot \ell+(1-p) \cdot \ell^{\prime \prime} \sim p \cdot \ell^{\prime}+(1-p) \cdot \ell^{\prime \prime}$;

6. if $p \cdot \ell+(1-p) \cdot \ell^{\prime \prime} \sim p \cdot \ell^{\prime}+(1-p) \cdot \ell^{\prime \prime}$, then $\ell \sim \ell^{\prime}$.

Definition 3. A preference relation is called linearly ordered (or linear, for short) if for every $\ell, \ell^{\prime} \in L$, either $\ell \preceq \ell^{\prime}$, or $\ell^{\prime} \preceq \ell$ (where $\ell \preceq \ell^{\prime}$ means that either $\ell \prec \ell^{\prime}$ or $\ell \sim \ell^{\prime}$ ).

It is known that linearly ordered preference relations can be characterized in terms of special functions called utility functions:

Definition 4. A function $u$ from the set $L$ of all lotteries to an ordered set $V$ is called a utility function. For each $\ell \in L, u(\ell)$ will be called a value of the utility function. We say that a utility function $u$ describes the preference relation if for every $\ell, \ell^{\prime} \in L$, the following two conditions hold:

$-\ell \prec \ell^{\prime}$ if and only if $u(\ell)<u\left(\ell^{\prime}\right)$;

$-\ell \sim \ell^{\prime}$ if and only if $u(\ell)=u\left(\ell^{\prime}\right)$.

Definition 5. A utility function $u: L \rightarrow V$ is called convexity-preserving if on the set $V$, convex combination $p_{1} \cdot v_{1}+\ldots+p_{n} \cdot v_{n}$ is defined for all $p_{i} \geq 0$, $\sum p_{i}=1$, and if for every $p_{i}$ and $\ell_{i}$, we have $u\left(p_{1} \cdot \ell_{1}+\ldots+p_{m} \cdot \ell_{m}\right)=$ $p_{1} \cdot u\left(\ell_{1}\right)+\ldots+p_{m} \cdot u\left(\ell_{m}\right)$. 
To describe linearly ordered preference relations, we use scalar utility functions, i.e., convexity-preserving utility functions for which $V=R$. It is known that for every convexity-preserving function $u: L \rightarrow R$, the relations $u(\ell)<u\left(\ell^{\prime}\right)$ and $u(\ell)=u\left(\ell^{\prime}\right)$ define a linearly ordered preference relation. It is also known that this utility function is determined uniquely modulo a linear transformation, i.e.:

- If two different scalar utility functions $u: L \rightarrow R$ and $u^{\prime}: L \rightarrow R$ describe the same preference relation, then there exists a linear function $T(z)=k \cdot z+m$, with $k>0$, such that for every lottery $\ell, u^{\prime}(\ell)=T(u(\ell))$.

- Vice versa, if a scalar utility function $u: L \rightarrow R$ describes a preference relation, and $k>0$ and $m$ are real numbers, then the function $u^{\prime}(\ell)=$ $T(u(\ell)$ ) (where $T(z)=k \cdot z+m$ ) is also a scalar utility function which describes the same preference relation.

One can also show that every Archimedean (in some reasonable sense) linearly ordered preference relation $\langle\prec, \sim\rangle$ can be described by an appropriate scalar utility function.

\section{New Approach: Utility Theory for Partially Ordered Preferences}

It turns out that a similar result holds for partially ordered references as well. To describe this result, we need to to recall a few definitions.

An affine space (see, e.g., [5] and references therein) is "almost" a vector space, the main difference between them is that in the linear space, there is a fixed starting point (0), while in the affine space, there is no fixed point. More formally:

- A linear space is defined as a set $V$ with two operations: addition $v+v^{\prime}$ and multiplication $\lambda \cdot v$ of elements from $V$ by real numbers $\lambda \in R$ (operations which must satisfy some natural properties). With this two basic operations, we can define an arbitrary linear combination $\lambda_{1} \cdot v_{1}+\ldots+\lambda_{n} \cdot v_{n}$ of elements $v_{1}, \ldots, v_{n} \in V$.

- In the affine space, we can only define those linear combination which are shift-invariant, i.e., linear combinations with $\sum \lambda_{i}=1$.

The relation between a linear space and an affine space is rather straightforward:

- if we have an affine space $V$, then we can pick an arbitrary point $v_{0} \in V$, are define a linear space in which this point is 0 . Namely, we can define $v+v^{\prime}$ as $1 \cdot v+1 \cdot v^{\prime}-1 \cdot v_{0}$ : since we took $v_{0}$ as 0 , this linear combination will be exactly $v+v^{\prime}$.

- Vice versa, if we have a hyperplane $H$ in a linear space, then (unless this hyperplane goes through 0) this hyperplane is not a linear space, but it is always an affine space. 
Definition 6. A vector space $V$ with a strict order $<$ is called an ordered vector space if for every $v, v^{\prime}, v^{\prime \prime} \in V$, and for every real number $\lambda>0$ the following two properties are true:

- if $v<v^{\prime}$, then $v+v^{\prime \prime}<v^{\prime}+v^{\prime \prime}$;

- if $v<v^{\prime}$, then $\lambda \cdot v<\lambda \cdot v^{\prime}$.

Since this ordering does not change under shift, it, in effect, defines an ordering on the affine space.

Definition 7. By a vector utility function, we mean a convexity-preserving utility function with values in an ordered affine space $V$.

To analyze uniqueness of vector utility functions, we must consider isomorphisms. A mapping $T$ between two affine spaces is called affine if it preserves the affine structure, i.e., if $T\left(\sum \lambda_{i} \cdot v_{i}\right)=\sum \lambda_{i} \cdot T\left(v_{i}\right)$ whenever $\sum \lambda_{i}=1$. For finite-dimensional affine spaces, affine mappings are just linear transformations $\left(x_{1}, \ldots, x_{n}\right) \rightarrow\left(y_{1}, \ldots, y_{m}\right)$, i.e., transformations in which each resulting coordinate $y_{i}$ is determined by a linear function $y_{i}=a_{i}+\sum b_{i j} \cdot x_{j}$.

Definition 8. A one-to-one affine transformation $T: V \rightarrow V^{\prime}$ of two ordered affine spaces is called an isomorphism if for every $v_{1}, v_{2} \in V, v<v^{\prime}$ if and only if $T(v)<T\left(v^{\prime}\right)$.

Recall that for every subset $S \subseteq V$ of an affine space, its affine hull $A(S)$ can be defined as the smallest affine subspace containing $S$, i.e., equivalently, as the set of all affine combinations $\sum \lambda_{i} \cdot s_{i}\left(\sum \lambda_{i}=1\right)$ of elements from $S$.

Theorem 1. Let $\mathcal{A}$ be a set, and let $L$ be the set of all lotteries over $\mathcal{A}$.

- (consistency) For every convexity-preserving function $u: L \rightarrow V$ from $L$ to an ordered affine space, the relations $u(\ell)<u\left(\ell^{\prime}\right)$ and $u(\ell)=u\left(\ell^{\prime}\right)$ define a preference relation.

- (existence) For every preference relation $\langle\prec, \sim\rangle$, there exists a vector utility function which describes this preference.

- (uniqueness) The utility function is determined uniquely modulo an isomorphism:

- If two different vector utility functions $u: L \rightarrow V$ and $u^{\prime}: L \rightarrow V^{\prime}$ describe the same preference relation, then there exists an isomorphism $T: A(u(L)) \rightarrow A\left(u^{\prime}(L)\right)$ between the affine hulls of the images of the functions, such that for every lottery $\ell, u^{\prime}(\ell)=T(u(\ell))$.

- Vice versa, if a vector utility function $u: L \rightarrow V$ describes a preference relation, and $T: A(u(L)) \rightarrow V^{\prime}$ is an isomorphism of ordered affine spaces, then the function $u^{\prime}(\ell)=T(u(\ell))$ is also a vector utility function, and it describes the same preference relation. 
Example. In the above tank example, it is natural to describe each possible damage outcome by a vector-valued utility $\left(u_{1}, u_{2}\right)$, where $u_{1}$ describes the tank's shooting abilities and $u_{2}$ the tank's moving abilities. This is, of course, a simplified example, we also need to take into consideration communication capabilities, possibility of damage repair, etc.--which leads to a higher-dimensional utility vector.

\section{How to Describe Degrees of Belief ("Subjective Probabilities") for Partially Ordered Preferences?}

In traditional (scalar) utility theory, it is possible to describe our degree of belief $p s(E)$ in each statement $E$, e.g., as follows: We pick two alternatives $a_{0}$ and $a_{1}$ with utilities 0 and 1 , and as the degree of belief in $E$, we take the utility of a conditional alternative "if $E$ then $a_{1}$ else $a_{0}$ " (or $\left(E\left|a_{1}\right| a_{0}\right)$, for short). This utility is also called subjective probability because if $E$ is a truly random event which occurs with probability $p$, then this definition leads to $p s(E)=p$ : Indeed, according to the convexity-preserving property of a utility function, we have

$$
p s(E)=u\left(E\left|a_{1}\right| a_{0}\right)=p \cdot u\left(a_{1}\right)+(1-p) \cdot u\left(a_{0}\right)=p \cdot 1+(1-p) \cdot 0=p .
$$

How can a similar description look like for partially ordered preferences? Before we formulate our result, let us first explain our reasoning that led to this result. The linear-ordered case definition of subjective probability $p s(E)$ can be rewritten as follows: for every two lotteries $\ell, \ell^{\prime} \in L$, we have

$$
u\left(E|\ell| \ell^{\prime}\right)=p s(E) \cdot u(\ell)+(1-p s(E)) \cdot u\left(\ell^{\prime}\right),
$$

or, equivalently,

$$
u\left(E|\ell| \ell^{\prime}\right)=p s(E) \cdot\left(u(\ell)-u\left(\ell^{\prime}\right)\right)+u\left(\ell^{\prime}\right) .
$$

In other words, we can interpret $p s(E)$ as a linear operator which transforms the utility difference $u(\ell)-u\left(\ell^{\prime}\right)$ into an expression

$$
u\left(E|\ell| \ell^{\prime}\right)-u\left(\ell^{\prime}\right)=p s(E) \cdot\left(u(\ell)-u\left(\ell^{\prime}\right)\right) .
$$

It is, therefore, reasonable to expect that for partially ordered preferences, when we have multi-dimensional (vector) utilities with values in a vector space $V$, $p s(E)$ would also be a linear operator, but this time from $V$ to $V$ (and not from $R$ to $R$ ). We will now show that this expectation is indeed true.

Definition 9. Let $\mathcal{A}$ be a set, let $L$ be the set of all lotteries over $\mathcal{A}$, and let $E$ be a formula (called event). By a conditional lottery, we mean an expression of the type $\sum p_{i} \cdot \ell_{i}+\sum q_{k} \cdot\left(E\left|\ell_{k}^{\prime}\right| \ell_{k}^{\prime \prime}\right)$, where $\sum p_{i}+\sum q_{k}=1$, and $\ell_{i}, \ell_{k}^{\prime}$, and $\ell_{k}^{\prime \prime}$ are lotteries. We will denote the set of all conditional lotteries by $L(E)$.

The meaning of a conditional lottery is straightforward: with probability $p_{i}$, we run a lottery $\ell_{i}$, and with probability $q_{k}$, we run a conditional event "if $E$ then $\ell_{k}^{\prime}$ else $\ell_{k}^{\prime \prime \prime}$. 
Definition 10. Let $\mathcal{A}$ be a set, and let $L(E)$ be the set of all conditional lotteries over $\mathcal{A}$. By a preference relation, we mean a pair $\langle\prec, \sim\rangle$, where $\prec$ is a (strict) order on $L(E), \sim i$ an equivalence relation on $L(E)$, which satisfies conditions 1)-6) from Definition 2 plus the following additional conditions:

1. if $\ell \sim \ell^{\prime}$, then $\left(E|\ell| \ell^{\prime \prime}\right) \sim\left(E\left|\ell^{\prime}\right| \ell^{\prime \prime}\right)$;

2. if $\ell^{\prime} \sim \ell^{\prime \prime \prime}$, then $\left(E|\ell| \ell^{\prime}\right) \sim\left(E|\ell| \ell^{\prime \prime}\right)$;

3. $(E|\ell| \ell) \sim \ell$;

4. $\left(E\left|p \cdot \ell+(1-p) \cdot \ell^{\prime}\right| \ell^{\prime \prime}\right) \sim p \cdot\left(E|\ell| \ell^{\prime \prime}\right)+(1-p) \cdot\left(E\left|\ell^{\prime}\right| \ell^{\prime \prime}\right)$;

5. $\left(E|\ell| p \cdot \ell^{\prime}+(1-p) \cdot \ell^{\prime \prime}\right) \sim p \cdot\left(E|\ell| \ell^{\prime}\right)+(1-p) \cdot\left(E|\ell| \ell^{\prime \prime}\right)$;

6. $\left(E\left|p \cdot \ell+(1-p) \cdot \ell^{\prime \prime}\right| p \cdot \ell^{\prime}+(1-p) \cdot \ell^{\prime \prime}\right) \sim p \cdot\left(E|\ell| \ell^{\prime}\right)+(1-p) \cdot \ell^{\prime \prime}$;

7. if $\ell \preceq \ell^{\prime}$, then $\ell \preceq\left(E|\ell| \ell^{\prime}\right) \preceq \ell^{\prime}$.

The meaning of all these conditions is straightforward; e.g., 7) means that $\left(E|\ell| \ell^{\prime}\right)$ is better (or of the same quality) than $\ell$ because in the conditional alternative, both possibilities $\ell$ and $\ell^{\prime}$ are at least as good as $A$.

In accordance with our Theorem 2 , the utility of such events can be described by a vector utility function.

Definition 11. Let $V$ be an ordered vector space.

- A linear operator $T: V \rightarrow V$ is called non-negative (denoted $T \geq 0$ ) if $x>0$ implies $V x \geq 0$.

- A linear operator $T$ is called a probability operator if both $T$ and $\mathbf{1}-T$ are non-negative (where $\mathbf{1}$ is a unit transformation $v \rightarrow v$ ).

\section{Theorem 2.}

- Let $u: L \rightarrow V$ be a vector utility function and let $T: V \rightarrow V$ be a strict probability operator. Then, a function $u^{*}: L(E) \rightarrow V$ defined as

$$
u^{*}\left(\sum_{i} p_{i} \cdot \ell_{i}+\sum_{k} q_{k} \cdot\left(E\left|\ell_{k}^{\prime}\right| \ell_{k}^{\prime \prime}\right)\right)=\sum_{i} p_{i} \cdot u\left(\ell_{i}\right)+\sum_{k} q_{k} \cdot u^{*}\left(E\left|\ell_{k}^{\prime}\right| \ell_{k}^{\prime \prime}\right),
$$

with $u^{*}\left(E|\ell| \ell^{\prime}\right)=T u(\ell)+(\mathbf{1}-T) u\left(\ell^{\prime}\right)$, is a vector utility function which describes a preference relation on $L(E)$.

- Let $\langle\prec, \sim\rangle$ be a preference relation on $L(E)$, and let $u: L(E) \rightarrow V$ be a vector utility function which describes this preference. Then, there exists a probability operator $T: A(u(L)) \rightarrow V$ for which

$$
u\left(E|\ell| \ell^{\prime}\right)=T u(\ell)+(\mathbf{1}-T) u\left(\ell^{\prime}\right)
$$

for all $\ell$ and $\ell^{\prime}$.

Thus, we get a generalization of subjective probabilities, from scalar values $p \in[0,1]$ (which, in our description, correspond to scalar matrices) to general linear probability operators.

In general, to describe a matrix, we need to describe its $n^{2}$ components, where $n$ is the dimension of the space $V$. It turns out that to describe matrices that represent probability operators, much fewer parameters are needed:

Theorem 3. Let $V$ be an $n$-dimensional ordered vector space. Then, the set of all probability operators is at most $n$-dimensional. 
Paul J. Tanenbaum et al.

Idea of the proof. An ordering relation in an $n$-dimensional space is characterized by a closed convex cone of all non-negative elements such that $V$ is a linear hull of the cone. This cone is convex hull of its extreme generators; thus, we can find $n$ generators $e_{i}$ that form a base of a linear space $V$. To describe $T$, it is sufficient to know $T\left(e_{i}\right)$ for all $i$. For each generator $e_{i} \geq 0$, the condition $0 \leq T\left(e_{i}\right) \leq e_{i}$ implies that $T\left(e_{i}\right)$ belongs to the same cone generator, i.e., that $T\left(e_{i}\right)=\lambda_{i} \cdot e_{i}$ for some real number $\lambda_{i} \geq 0$. So, to describe $T$, it is enough to know $n$ values $\lambda_{i}$. Q.E.D.

Comment. Other results show that for most ordered vector spaces, we need even fewer parameters.

\section{Acknowledgments}

This work was supported in part by the Army Research Laboratory grant DATM-05-02-C-0046, by NASA grant NCC5-209, by Air Force Office of Scientific Research grant F49620-00-1-0365, by NSF grants EAR-0112968 and EAR0225670, by IEEE/ACM SC2001 and SC2002 Minority Serving Institutions Participation Grants, and by a research grant from Sandia National Laboratories as part of the Department of Energy Accelerated Strategic Computing Initiative (ASCI).

The authors are thankful to Peter C. Fishburn for valuable discussions and encouragement, and to the anonymous referees for valuable suggestions.

\section{References}

1. Bowers, R.A., Parker, R.G., Tanenbaum, P.J.: Tcl/Tk in Survivability Modeling for Military Systems, Proceedings of 8th Annual Tcl/Tk Conference, 2001.

2. Fishburn, P.C.: Utility theory for decision making, Wiley, New York (1969)

3. Fishburn, P.C.: Nonlinear preferences and utility theory, John Hopkins University Press, Baltimore (1988)

4. Fuchs, L.: Partially Ordered Algebraic Systems, Pergamon Press, New York (1963)

5. Itô, K. (ed.): Encyclopedic dictionary of mathematics, MIT Press, Cambridge, MA (1993)

6. Kosheleva, O.M., Kreinovich, V.: Computational complexity of game-theoretic problems, Technical Report, Leningrad Center for New Information Technology "Informatika", Leningrad (1989) in Russian

7. Kosheleva, O., Kreinovich, V., Nguyen, H.T., Bouchon-Meunier, B.: How to describe partially ordered preferences: mathematical foundations, In: Nguyen, H.P., Ohsato, A. (eds.): Proceedings of the Vietnam-Japan Bilateral Symposium on Fuzzy Systems and Applications VJFUZZY'98, HaLong Bay, Vietnam (1998) 269278; see http://www.cs.utep.edu/vladik/1998/tr98-23.ps.gz or -23.pdf

8. Markov, S.: On the Algebra of Intervals and Convex Bodies, J. UCS 4, No. 1 (1998) $34-47$

9. Markov, S.: On the Algebraic Properties of Convex Bodies and Some Applications, J. Convex Analysis 7 (2000) 129-166 